\title{
Molecular Abundances in the Envelopes Around Evolved Stars
}

\author{
Hans Olofsson
}

Onsala Space Observatory, S-43992 Onsala, Sweden

\section{Introduction}

Red giant stars on the asymptotic giant branch (AGB), AGB-stars, lose copious amounts of matter in a slow stellar wind (Olofsson 1993). Mass loss rates in excess of $10^{-4} \mathrm{M}_{\odot} \mathrm{yr}^{-1}$ have been measured. The primary observational consequence of this mass loss is the formation of an expanding envelope of gas and dust, a circumstellar envelope (CSE), that surrounds the star. This is a truly extended atmosphere that continues thousands of stellar radii away from the star. At the highest mass loss rates (which probably occur at the end of the AGB evolution) the CSE becomes so opaque that the photosphere is hidden and essentially all information about the object stems from the circumstellar emission. At some point on the AGB a star may change from being $\mathrm{O}$-rich (i.e., the abundance of $\mathrm{O}$ is higher than that of $\mathrm{C}$ ) to becoming $\mathrm{C}$-rich (i.e., a carbon star where the abundance of $\mathrm{C}$ is higher than that of $\mathrm{O}$ ) as a result of nuclear-processed material being dredged up to the surface. The chemical composition of the CSE will follow that of the central star, although with some time delay so that there may be some rare cases of $\mathrm{O}$-rich CSEs around carbon stars. The mass loss decreases and changes its nature as the star leaves the AGB and starts its postAGB evolution. Eventually the star becomes hot enough to ionize the inner part of the AGB-CSE and a planetary nebula (PN) is formed. The ultimate fate of the star is a long life as a slowly cooling white dwarf. The CSE will gradually disperse and its metal-enriched matter will mix with the interstellar medium, and thereby it contributes to the chemical evolution of a galaxy. The intense mass loss makes it possible for stars as massive as $8 \mathbf{M}_{\odot}$, i.e., the bulk of all stars in a galaxy, to follow this evolutionary sequence. Similar CSEs are also found around supergiants.

The AGB-CSEs form relatively well defined astrophysical laboratories. They appear to have an over-all spherical geometry. The gas expansion velocity is roughly constant throughout the envelope and it is typically of the order 10 to $15 \mathrm{~km} \mathrm{~s}^{-1}$. The gas density falls as $r^{-2}$, where $r$ is the radius, if the mass loss rate and expansion velocity are constant with time and radius, respectively, i.e., the number density spans quite a range within a CSE. The kinetic temperature 
is determined mainly by adiabatic cooling as a consequence of the expansion and heating due to gas-grain collisions as the dust grains stream through the gas, but there is also an additional cooling contributed by line radiation and there are other possible heating sources (see e.g., Truong-Bach et al. 1990). The inner region of a CSE may have temperatures as high as $1000 \mathrm{~K}$, while the external teneous parts may be as cool as $10 \mathrm{~K}$ or less. The molecules are exposed to radiation from two sources. First, radiation from the central star which has characteristics, depending on the evolutionary stage, that fall somewhere in the range from a luminous $\left(10^{4} \mathrm{~L}_{\odot}\right)$, cool $(2500 \mathrm{~K})$ red giant to a much less luminous $\left(<10^{2} \mathrm{~L}_{\odot}\right)$, but hot $(>30000 \mathrm{~K})$ star on its way to becoming a white dwarf. Second, the interstellar radiation field which is relatively isotropic, but there may be local anisotropies.

\section{Circumstellar molecules detected at radio wavelengths}

The first circumstellar molecule to be detected at radio wavelengths was $\mathrm{OH}$ in 1968 , i.e., 25 years ago (Wilson \& Barrett 1968). Initially the progress was slow, but in 1971 Solomon et al. (1971) detected CO from an infrared bright object, IRC +10216 . This is probably the most nearby carbon star, and it happens to have a very high mass loss rate, of the order a few $10^{-5} \mathrm{M}_{\odot} \mathrm{yr}^{-1}$ (Troung-Bach et al. 1992). It was quickly realized that a CSE around an evolved star, in particular a C-rich one, can be extremely rich in different molecular species. The reason being that the circumstellar dust effectively shields the molecules that are formed both in the stellar atmosphere and in the CSE itself, and in the $\mathrm{C}$-rich case the carbon excess leads to an efficient organic chemistry. At this epoch 45 circumstellar molecules have been detected at radio wavelengths, of these 38 in the envelope of IRC+10216, Table 1. Indeed, some of them (16) are only detected in IRC+10216, and most of them (31) in fewer than five sources. The fact that so few sources completely dominate the picture means that most of what will be dicussed below is based on meagre statistics.

Among the most interesting of these circumstellar molecules are the long carbon-chain ones, the cyanopolyynes $\mathrm{HC}_{n} \mathrm{~N}(n=3,5,7,9,11)$, the related hydrocarbons $\mathrm{C}_{n} \mathrm{H}(n=2,3,4,5,6)$ and $\mathrm{H}_{2} \mathrm{C}_{n}(n=3,4)$, as well as $\mathrm{HC}_{2} \mathrm{~N}$. Also nitrogen-carbon chains $\mathrm{C}_{n} \mathrm{~N}(n=1,3)$, silicon-carbon chains $\mathrm{C}_{n} \mathrm{Si}(n=1,4)$, and sulphur-carbon chains $\mathrm{C}_{n} \mathrm{~S}(n=1,2,3)$ are present. Simple ring molecules, like the triangular molecules $\mathrm{SiC}_{2}$ and cyclic- $\mathrm{C}_{3} \mathrm{H}_{2}$, have been detected, but branched molecules are notably absent. On the more spectacular side we have the detections of the refractory species $\mathrm{NaCl}, \mathrm{AlCl}, \mathrm{AlF}$, and $\mathrm{KCl}$ (Cernicharo \& Guélin 1987; Lucas \& Guélin 1990), and most recently the identification of three lines towards IRC+10216 as due to MgNC (Kawaguchi et al. 1993). The $\mathrm{O}$-rich CSEs are apparently less rich in molecules. Here $\mathrm{H}_{2} \mathrm{O}$ and $\mathrm{OH}$ dominate, but also sulphur-bearing species, in particular $\mathrm{SO}, \mathrm{SO}_{2}$, and $\mathrm{H}_{2} \mathrm{~S}$, are frequently found (Sahai \& Wannier 1992; Omont et al. 1993). 
Table 1. Circumstellar molecules detected at radio wavelengths

\begin{tabular}{|c|c|c|c|}
\hline Molecule & No. of objects & Molecule & No. of objects \\
\hline$\overline{\mathrm{AlCl}}$ & 1 & $\overline{\mathrm{OCS}}$ & 1 \\
\hline $\mathrm{AlF}$ & 1 & $\mathrm{SiC}_{2}$ & 4 \\
\hline $\mathrm{CO}$ & $\approx 420$ & $\mathrm{SO}_{2}$ & 17 \\
\hline $\mathrm{CN}$ & 17 & $\ell-\mathrm{C}_{3} \mathrm{H}$ & 2 \\
\hline $\mathrm{CP}$ & 1 & $\mathrm{C}_{3} \mathrm{~N}$ & 5 \\
\hline $\mathrm{CS}$ & 21 & $\mathrm{C}_{3} \mathrm{~S}$ & 1 \\
\hline $\mathrm{KCl}$ & 1 & $\mathrm{HC}_{2} \mathrm{~N}$ & 1 \\
\hline $\mathrm{NaCl}$ & 1 & $\mathrm{H}_{2} \mathrm{CO}$ & 2 \\
\hline $\mathrm{OH}$ & $>1500$ & $\mathrm{NH}_{3}$ & 4 \\
\hline $\mathrm{SiC}$ & 2 & $\mathrm{C}_{4} \mathrm{H}$ & 5 \\
\hline $\operatorname{SiN}$ & 1 & $\mathrm{C}_{4} \mathrm{Si}$ & 1 \\
\hline $\mathrm{SiO}$ & $\approx 50($ th $), \approx 200$ (maser) & $c-\mathrm{C}_{3} \mathrm{H}_{2}$ & 7 \\
\hline $\mathrm{SiS}$ & 6 & $\mathrm{HC}_{3} \mathrm{~N}$ & 7 \\
\hline $\mathrm{SO}$ & 9 & $\mathrm{H}_{2} \mathrm{C}_{3}$ & 1 \\
\hline $\mathrm{C}_{2} \mathrm{H}$ & 4 & $\mathrm{C}_{5} \mathrm{H}$ & 1 \\
\hline $\mathrm{C}_{2} \mathrm{~S}$ & 4 & $\mathrm{CH}_{3} \mathrm{CN}$ & 3 \\
\hline $\mathrm{HCN}$ & $\approx 110$ & $\mathrm{H}_{2} \mathrm{C}_{4}$ & 1 \\
\hline $\mathrm{HCO}^{+}$ & 7 & $\mathrm{C}_{6} \mathrm{H}$ & 1 \\
\hline $\mathrm{H}_{2} \mathrm{O}$ & $\approx 400$ & $\mathrm{HC}_{5} \mathrm{~N}$ & 4 \\
\hline $\mathrm{H}_{2} \mathrm{~S}$ & 20 & $\mathrm{HC}_{7} \mathrm{~N}$ & 2 \\
\hline $\mathrm{HNC}$ & 10 & $\mathrm{HC}_{9} \mathrm{~N}$ & 1 \\
\hline $\mathrm{MgNC}$ & 1 & $\mathrm{HC}_{11} \mathrm{~N}$ & 1 \\
\hline $\mathrm{NH}_{2}^{+}$ & 1 & & \\
\hline
\end{tabular}

\section{The nature of the circumstellar molecular radio line emission}

In order to derive reliable molecular abundances from the line emission one requires "well behaved" emission, i.e., one tries to avoid emission from transitions that are anomalously excited. The extreme example of the latter is maser emission, i.e., amplified line radiation due to a transition with a population inversion, which unfortunately is not an uncommon phenomenon in the circumstellar medium (Elitzur 1992). We will not give an exhaustive discussion of this problem, but rather in brief, state our current knowledge.

There is strong maser emission from the ${ }^{2} \Pi_{3 / 2}, J=3 / 2 \Lambda$-doubling transitions of $\mathrm{OH}$ (Cohen 1989), from several rotational transitions and one vibrationally excited rotational line of $\mathrm{H}_{2} \mathrm{O}$ (Menten \& Melnick 1989, 1991; Cernicharo et al. 1990; Menten et al. 1990; Lewis \& Engels 1991), from several vibrationally excited rotational lines of $\mathrm{SiO}$ and also the rare isotope variants ${ }^{29} \mathrm{SiO}$ and ${ }^{30} \mathrm{SiO}$ (Cernicharo \& Bujarrabal 1992; Cernicharo et al. 1993), and at least two vibrationally excited rotational transitions of HCN (Guilloteau et al. 1987; Lucas \& Cernicharo 1989). This means that in the case of $\mathrm{OH}$ and $\mathrm{H}_{2} \mathrm{O}$, where no nonmasing lines have been detected, there exists no observational estimate of their circumstellar abundances. Weak maser emission from vibrationally excited ro- 
tational lines are found towards IRC+10216 for CS (Turner 1987a), SiS (Turner 1987b), and HCN (Lucas \& Cernicharo 1989). Strong maser emission is usually easy to identify because of the complex line profile and/or the strong time variability.

Maser emission from ground-state transition lines is more of a problem since these are normally the ones used for estimating abundances. Fortunately, in most cases this maser emission contributes to only a minor fraction of the total line intensity. Weak maser emission has been found in the $\operatorname{HCN}(J=1-0)$ line towards tenuous, C-rich CSEs (Izumiura, 1990; Olofsson et al. 1993b), and the same applies to the $\mathrm{H}^{13} \mathrm{CN}(J=1-0)$ line although here the statistics is poor (Izumiura et al. 1987; Nyman et al. 1993). The frequently observed $\mathrm{SiO}(J=2$ 1) line only occasionally shows evidence for weak maser features (Nyman \& Olofsson 1985), while the less frequently observed $\mathrm{SiO}(J=1-0)$ line appears to be more affected by maser emission (Jewell et al. 1991). Surprisingly, strong maser emission has been found for ${ }^{29} \mathrm{SiO}$ and ${ }^{30} \mathrm{SiO}$ in the $J=1-0,2-1$, and $5-4$ lines (Alcolea \& Bujarrabal 1992). The CS( $J=2-1)$ line shows evidence for a maser feature in one case (Nyman et al. 1993). The SiS $(J=1-0)$ line of IRC+10216 shows relatively strong maser components (Nguyen-Q-Rieu et al. 1984), and the $\operatorname{SiS}(J=5-4)$ line has a remarkable behaviour towards the same object that may indicate contributions from maser emission (Carlström et al. 1990).

That is, maser emission is a surprisingly common phenomenon, and even though its contribution to the total line intensity may be small, it does show that we can in general expect an excitation that is far from LTE.

\section{Circumstellar molecular abundances: theory}

\subsection{Abundance formula}

In principle, a fairly detailed modelling is required, which in some cases includes a complex non-local radiative transfer analysis (see e.g., Truong-Bach \& NguyenQ-Rieu 1989), to obtain the abundance of a circumstellar molecule. However, for most molecules in Table 1 this is a too cumbersome approach, e.g., radiative transition rates and collisional cross sections are missing, and a simpler method is usually adopted. The model is based on the assumption that a spherical CSE of gas, expanding at a constant velocity, $v_{\mathrm{e}}$, is formed by a constant stellar mass loss rate $\dot{M}$. The molecules are thermally excited at a temperature $T_{\text {ex }}$ inside a shell of inner radius $r_{\mathrm{i}}$ and outer radius $r_{\mathrm{e}}$. Molecules outside this shell do not contribute to the emission. The response of the telescope is assumed to be described by a Gaussian defined by the FWHM of the beam, $B$. Reasonably reliable estimates of molecular abundances are only obtained using optically thin lines, and in this limit (and in the Rayleigh-Jeans regime) the abundance of the molecule $\mathrm{X}$ with respect to $\mathrm{H}_{2}, f_{\mathrm{X}}$, is obtained using (see e.g., Olofsson et al. 1993b)

$$
f_{\mathrm{X}}=1.7 \times 10^{-28} I \frac{v_{\mathrm{e}} B D}{\dot{M}_{\mathrm{H}_{2}}} \frac{Q\left(T_{\mathrm{ex}}\right) \nu_{u l}^{2}}{g_{u} A_{u l}} \frac{e^{E_{1} / k T_{\mathrm{ex}}}}{\int_{x_{\mathrm{i}}}^{x_{\mathrm{e}}} e^{-4 \ln 2 x^{2} d x}}
$$


where $I$ is the line intensity integrated over velocity in units of $\mathrm{K} \mathrm{km} \mathrm{s}^{-1}, v_{\mathrm{e}}$ is given in $\mathrm{km} \mathrm{s}^{-1}, B$ in arc seconds, $D$ is the distance to the source in $\mathrm{pc}$, $\dot{M}_{\mathrm{H}_{2}}$ is the mass loss rate of molecular hydrogen [the assumption that the major constituent of hydrogen in CSEs is molecular hydrogen is usually justified, Glassgold \& Huggins (1983)] given in $\mathrm{M}_{\odot} \mathrm{yr}^{-1}, Q$ is the partition function, $\nu_{u l}$ is the frequency in $\mathrm{GHz}, g_{u}$ is the degeneracy of the upper level, $A_{u l}$ is the Einstein $A$-coefficient for the transition, $E_{l}$ is the energy of the lower level, and $x=r / B D$ and $x_{\mathrm{i}, \mathrm{e}}=r_{\mathrm{i}, \mathrm{e}} / B D$. Usually the emitting region is smaller than the beam and we may simplify further to get

$$
f_{\mathrm{X}}=2.5 \times 10^{-15} I \frac{v_{\mathrm{e}} B^{2} D^{2}}{\dot{M}_{\mathrm{H}_{2}}} \frac{Q\left(T_{\mathrm{ex}}\right) \nu_{u l}^{2}}{g_{u} A_{u l}} \frac{e^{E_{l} / k T_{\mathrm{ex}}}}{r_{\mathrm{e}}-r_{\mathrm{i}}}
$$

where $r_{\mathrm{i}}$ and $r_{\mathrm{e}}$ are given in $\mathrm{cm}$. Essentially all reported circumstellar molecular abundances are based on Eq.(2) or variants thereof, see Sect. 4.3.

The molecular abundance estimate, obtained using Eq.(1) or Eq.(2), is critically dependent on such uncertain quantities as $T_{\mathrm{ex}}, D, \dot{M}_{\mathrm{H}_{2}}, r_{\mathrm{i}}$, and $r_{\mathrm{e}}$, and we proceed now to discuss these.

\subsection{The source size}

The size of the emitting region, i.e., $r_{\mathrm{i}}$ and $r_{\mathrm{e}}$, is crucial in the calculation of the molecular abundance. Unfortunately, it has been measured only for a few sources and for a very limited number of molecular species. Additionally, the size is dependent on the particular transition in question. In principle, the only data with reasonable statistics are those on $\mathrm{SiO}$ (Lucas et al. 1992; Sahai \& Bieging 1993), and $\mathrm{OH}$ (Bowers et al. 1980, 1983). For a number of species, e.g., SiS, $\mathrm{HCN}, \mathrm{HNC}, \mathrm{HC}_{3} \mathrm{~N}, \mathrm{C}_{2} \mathrm{H}, \mathrm{C}_{4} \mathrm{H}, \mathrm{C}_{3} \mathrm{~N}$, and $\mathrm{SiC}_{2}$, there exist high-quality maps for only a single object, IRC+10216 (Bieging et al. 1984; Bieging \& Nguyen-Q-Rieu 1988, 1989; Gensheimer et al. 1992; Takano et al. 1992; Bieging \& Tafalla 1993; Dayal \& Bieging 1993). Thus, in most cases the size of the emitting region has to be estimated in a different way.

The initial circumstellar abundance of a parent species is determined by the upper stellar atmosphere chemistry, $f_{\mathrm{X}}\left(R_{*}\right)$. The abundance remains at this value until some point in the envelope where the interstellar UV photon density becomes high enough to dissociate the molecules. The location of this point is determined by the effectiveness of the dust shielding and whether the molecule is self-shielding, i.e., whether it is photodissociated in lines, or through continuous absorption. The abundance as a function of radius is determined by (Huggins \& Glassgold 1982)

$$
\frac{d f_{\mathrm{X}}}{d r}=-\frac{G_{0, \mathrm{X}}}{v_{\mathrm{e}}} \exp \left(-\frac{d\left(N_{\mathrm{X}}, \tau_{\mathrm{d}}\right)}{r}\right)
$$

where $G_{0, \mathrm{X}}$ is the unshielded photodissociation rate for a given interstellar UV field, and the shielding distance $d\left(N_{\mathrm{X}}, \tau_{\mathrm{d}}\right)$ is dependent on the outward dust optical depth $\tau_{\mathrm{d}}$, and, if self-shielding dominates, the outward column density $N_{\mathrm{X}}$ of the molecule in question (for some self-shielding molecules there is an 
additional shielding due to line absorption from other molecules, e.g., $\mathrm{H}_{2}$ helps shielding $\mathrm{CO}$ ). The shielding distance for pure dust absorption is given by (see e.g., Olofsson et al. 1993b)

$$
d_{\mathrm{X}}=1.4 \frac{3\left(Q_{\mathrm{abs}} / a\right)_{\mathrm{X}}}{4 \rho_{\mathrm{d}}} \frac{\dot{M}_{\mathrm{d}}}{4 \pi v_{\mathrm{d}}}
$$

where $Q_{\text {abs }}$ is the dust absorption efficiency, $a$ is the size of a dust grain and $\rho_{\mathrm{d}}$ its density, $\dot{M}_{\mathrm{d}}$ is the dust mass loss rate, and $v_{\mathrm{d}}$ the dust expansion velocity (the factor 1.4 approximately takes into account the three-dimensionality of the problem). By far, the largest molecular envelopes are found for the self-shielding species $\mathrm{CO}$ (Mamon et al. 1988) and $\mathrm{H}_{2}$ (Glassgold \& Huggins 1983). The result for $\mathrm{CO}$ can be approximated by

$$
R_{\mathrm{e}}(\mathrm{CO})=10^{17}\left(\frac{\dot{M}}{10^{-6} \mathrm{M}_{\odot} \mathrm{yr}^{-1}}\right)^{0.6}\left(\frac{15 \mathrm{~km} \mathrm{~s}^{-1}}{v_{\mathrm{e}}}\right)^{0.4}\left(\frac{f_{\mathrm{CO}}}{10^{-3}}\right)^{0.5} \mathrm{~cm} .
$$

For comparison, a mass loss rate of $10^{-5} \mathrm{M}_{\odot} \mathrm{yr}^{-1}$ leads to $R_{\mathrm{e}}(\mathrm{CO}) \approx 4 \times 10^{17} \mathrm{~cm}$ while $R_{\mathrm{e}}(\mathrm{HCN}) \approx 10^{16} \mathrm{~cm}$ (Olofsson et al. 1993b). For parent species we have $r_{\mathrm{i}} \ll r_{\mathrm{e}}$ and $r_{\mathrm{e}} \leq R_{\mathrm{e}}\left(r_{\mathrm{e}}\right.$ is the outer radius of the brightness distribution in the simple model discussed in Sect. 4.1). The exact value of $r_{\mathrm{e}}$ depends on the molecule and the mass loss rate, i.e., whether the emitting region is photodissociation limited or brightness limited. In the former case one usually chooses $r_{\mathrm{e}}$ as the $e$-folding radius, i.e., such that $f_{\mathrm{X}}\left(r_{\mathrm{e}}\right)=f_{\mathrm{X}}\left(R_{*}\right) / e$. Typical examples of parent species are $\mathrm{CO}, \mathrm{SiO}, \mathrm{HCN}$ (in $\mathrm{C}$-rich objects), and $\mathrm{H}_{2} \mathrm{~S}$.

The abundance of a photodissociation product is initially very low, but it increases at the radius where the parent species $X_{p}$ becomes photodissociated. A peak is reached since eventually the photodissociation product also becomes photodissociated, i.e., these molceules are distributed in a shell. The abundance as a function of radius is given by (Huggins \& Glassgold 1982)

$$
\frac{d f_{\mathrm{X}}}{d r}=-\frac{G_{0, \mathrm{X}}}{v_{\mathrm{e}}} \exp \left(-\frac{d\left(N_{\mathrm{X}}, \tau_{\mathrm{d}}\right)}{r}\right)+\frac{G_{0, \mathbf{X}_{\mathrm{p}}}}{v_{\mathrm{e}}} \exp \left(-\frac{d\left(N_{\mathbf{X}_{\mathrm{p}}}, \tau_{\mathrm{d}}\right)}{r}\right)
$$

In the event that the brightness distribution is photodissociation limited one usually chooses $r_{\mathrm{i}}$ and $r_{\mathrm{e}}$ as the inner and outer radii where the abundance has dropped to $f_{\mathrm{X} \text {,peak }} / e$. Typical examples of photodissociation products are $\mathrm{CN}$, $\mathrm{C}_{2} \mathrm{H}$, and $\mathrm{C}_{3} \mathrm{~N}$.

This model for circumstellar photodissociation has a number of weaknesses. The three dimensional geometry of the problem is often treated in a relatively crude way (Jura \& Morris 1981). Only dust absorption is taken into account, and even so the absorbing properties, as well as the actual amount, of the dust particles are poorly known. The effectiveness of the self-shielding is dependent on the kinematics of the molecules in the external parts of the CSE. The interstellar UV radiation field is highly uncertain and may vary from place to place, and in certain locations it may even be anisotropic (van Dishoeck 1988, and references therein). In general, while the photodissociation coefficients are well determined 
for some species like $\mathrm{H}_{2}, \mathrm{CO}, \mathrm{H}_{2} \mathrm{O}$, and $\mathrm{OH}$, they are poorly known for many molecules (see e.g., van Dishoeck 1988). We have also ignored the possibility that the photodissociation product of the parent species rapidly reacts chemically with another species. However, the major error source may be the actual density distribution of the molecules in the CSE. In a highly clumped medium, for which there is growing evidence (Olofsson et al. 1992; Bieging \& Tafalla, 1993), the molecules may survive to a much bigger distance from the star than given by the above model, which is based on the assumption of a homogeneous density distribution (Bergman et al. 1993).

Finally, there are molecules whose existence depends on a circumstellar chemistry initiated by the photodissociation processes (see e.g., Millar 1988). Their radial distributions are more difficult to predict since they depend on a complicated mixture of chemical reactions. For instance, the ionization of acetylene leads to a complex ion-molecule chemistry in C-rich CSEs (Nejad \& Millar 1987; Howe \& Millar 1990; Glassgold et al. 1992). Neutral-neutral reactions may be responsible for the production of some carbon-chain molecules (Howe \& Millar 1990). Likewise, the photodissociation of $\mathrm{H}_{2} \mathrm{O}$ in O-rich CSEs produces $\mathrm{OH}$ which rapidly reacts to form other species like $\mathrm{SO}$ and $\mathrm{SO}_{2}$ (Scalo \& Slavsky 1980). Another interesting example concerns the production of C-bearing species in O-rich CSEs (Nejad \& Millar 1988; Nercessian et al. 1989). In the latter cases the chemistry is of the neutral-neutral type and it becomes critically dependent on the existence of activation energy barriers and therefore becomes particularly uncertain. The actual choice of $r_{\mathrm{i}}$ and $r_{\mathrm{e}}$ therefore depends on relatively complex models. It is probably true that the majority of the molecules in Table 1 is due to a circumstellar chemistry. For some species we have the additional problem that grain surface reactions may be of importance. Typical examples of this kind are $\mathrm{CH}_{4}$ (Clegg et al. 1982) and $\mathrm{SiH}_{4}$ (Betz 1987).

In summary, the dependence of the estimated molecular abundance on $r_{\mathrm{i}}$ and $r_{\mathrm{e}}$ will lead to substantial uncertainties. In future, the situation is likely to improve considerably as more and more source sizes are actually measured observationally.

\subsection{The excitation temperature}

The discussion in Sect. 3 implies that a relatively complex non-LTE excitation analysis is required to obtain the excitation temperature and the correct partition function. However, at present it seems that such a detailed analysis for each molecular species is not warranted considering all other uncertainties in the circumstellar model, and in many cases it is not even possible. A similar treatment of all data based on the simplifying assumption of a uniform excitation temperature still seems appropriate. The excitation temperature $T_{\text {ex }}$ can be estimated in the following way by observing two or more optically thin lines from the same species. Equation (1) may be rewritten in the form

$$
\ln \left(\frac{(I / \eta) \nu_{u l}^{2}}{g_{u} A_{u l}}\right)+\ln \left(1.8 \times 10^{10} \frac{Q\left(T_{\mathrm{ex}}\right)}{\vec{N}_{\mathrm{X}}}\right)=-\frac{E_{l}}{k T_{\mathrm{ex}}}
$$


where $\eta=\int_{x_{\mathrm{i}}}^{x_{e}} e^{-4 \ln 2 x^{2}} d x$ and $\bar{N}_{\mathrm{X}}$ is the beam-averaged column density defined by

$$
\bar{N}_{\mathrm{X}}=\frac{f_{\mathrm{X}} \dot{M}_{\mathrm{H}_{2}}}{8 \pi m_{\mathrm{H}} v_{\mathrm{e}} B D}
$$

and it is given in $\mathrm{cm}^{-2}$, and $I$ is given in $\mathrm{K} \mathrm{km} \mathrm{s}^{-1}$, and $\nu_{u l}$ in $\mathrm{GHz}$. For two arbitrary lines this results in

$$
T_{\mathrm{ex}}=\frac{E_{l 2}-E_{l 1}}{k} \ln \left(\frac{g_{u 2} A_{u l 2} I_{1}}{g_{u 1} A_{u l 1} I_{2}}\right) .
$$

It is assumed that the emitting regions of the two lines are the same (this is a questionable assumption in many cases, since one expects a line from high-lying energy levels to emanate from a smaller area than a line from low-lying energy levels). Furthermore, they must be unresolved by the telescope beam. We have also used the fact that the beam size is inversely proportional to the frequency (i.e., both lines are observed with the same telescope). A statistically more reliable value for the excitation temperature is obtained by combining data from many transitions in a rotation temperature diagram where $\ln \left((I / \eta) \nu_{u l}^{2} / g_{u} A_{u l}\right)$ is plotted versus $E_{l} / k$ (see e.g., Cernicharo et al. 1987b). A fit of a straight line to the data in such a diagram, $y=\alpha+\beta E_{l} / k$, leads to an estimate of the excitation temperature

$$
T_{\mathrm{ex}}=-\frac{1}{\beta}
$$

and also of the beam averaged column density

$$
\bar{N}_{\mathrm{X}}=1.8 \times 10^{10} Q\left(T_{\mathrm{ex}}\right) e^{\alpha}
$$

which is an alternative way of expressing the molecular abundance. In fact, this gives a statistically more reliable abundance estimate than that obtained from Eq.(1) or Eq.(2) which is based on a single line.

However, in most cases a reasonable value for $T_{\text {ex }}$ is simply assumed, and in the worst case this is a pure guess. This is particularly unfortunate since the dependence of $f_{\mathrm{X}}$ on $T_{\mathrm{ex}}$ is quite strong, roughly $T_{\mathrm{ex}} \exp \left(E_{l} / k T_{\mathrm{ex}}\right)$ for linear rotors and $T_{\mathrm{ex}}^{3 / 2} \exp \left(E_{l} / k T_{\mathrm{ex}}\right)$ for symmetric and asymmetric top molecules.

\subsection{The mass loss rate and the distance}

The mass loss rate is often highly uncertain (van der Veen \& Olofsson 1990; Olofsson 1993). It is not unrealistic to expect an uncertainty that approaches an order of magnitude in many cases. This quantity enters into the calculation of the abundance not only through $\dot{M}_{\mathrm{H}_{2}}$ but also via the size of the emitting region since the photodissociation radius, the chemistry, and the excitation are all dependent on it. The combined effect is that the mass loss rate of the star is probably the major error source in the abundance estimate. In fact, it can be concluded from studies of circumstellar HCN and CS (Olofsson et al. 1993b), 
and $\mathrm{SO}$ and $\mathrm{SO}_{2}$ (Sahai \& Wannier 1992), that the mass loss rates of the stars in their samples are probably significantly higher than the accepted values.

The distances to the stars of interest here, are notoriously uncertain, but often the mass loss rate estimate has a dependence on distance which is close to $D^{2}$ and this significantly decreases the dependence of $f_{\mathrm{X}}$ on distance.

\subsection{A note on the column density}

Interstellar molecular abundances are frequently given in terms of column densities, i.e., the number of molecules within a column of given cross section. Occasionally, this is also done in the circumstellar case, but the relation between column density and abundance is not straightforward. If the mass loss rate, and the gas expansion velocity and the molecular abundance are assumed to be constant with time and radius, respectively, then the molecular density varies as

$$
n_{\mathrm{X}}(r)=n_{\mathrm{X}} R_{\mathrm{o}}^{2} \frac{1}{r^{2}}
$$

where $n_{\mathrm{Xo}}$ is the number density of species $\mathrm{X}$ at the arbitrary radius $R_{\mathrm{O}}$, and $n_{\mathrm{X}} R_{\mathrm{o}}^{2}$ is related to the abundance $f_{\mathrm{X}}$ via

$$
n_{\mathrm{Xo}} R_{\circ}^{2}=\frac{f_{\mathrm{X}} \dot{M}_{\mathrm{H}_{2}}}{8 \pi m_{\mathrm{H}} v_{\mathrm{e}}}
$$

The column density $N_{\mathrm{X}}$ towards the star is given by

$$
N_{\mathrm{X}}=n_{\mathrm{Xo}} R_{\circ}^{2}\left(\frac{1}{R_{\mathrm{i}}}-\frac{1}{R_{\mathrm{e}}}\right) \approx n_{\mathrm{Xo}} R_{\mathrm{o}}^{2} \frac{1}{R_{\mathrm{i}}}
$$

where $R_{\mathrm{i}}$ and $R_{\mathrm{e}}$ are the inner and outer radii of the molecular distribution (note that $R_{\mathrm{i}}$ and $R_{\mathrm{e}}$ are not necessarily the same as $r_{\mathrm{i}}$ and $r_{\mathrm{e}}$ ). The column density and the abundance are consequently related through

$$
N_{\mathrm{X}}=\frac{f_{\mathrm{X}} \dot{M}_{\mathrm{H}_{2}}}{8 \pi m_{\mathrm{H}} v_{\mathrm{e}}} \frac{1}{R_{\mathrm{i}}}
$$

i.e., via the observationally ill-defined quantity $R_{\mathrm{i}}$. Infrared absorption line measurements naturally lead to $N_{\mathrm{X}}$, while the radio observations are dependent on the number of molecules within the beam, and hence are better defined by the abundance $f_{\mathrm{X}}$ or the beam averaged column density $\bar{N}_{\mathrm{X}}$, defined in Eq.(8). It is apparent from this discussion that a comparison between radio and infrared data is not straightforward, nor is the comparison between radio data taken with different beam sizes. 


\section{Circumstellar molecular abundances: observational estimates}

We have in Table 2 summarised a number of molecular abundances in the CSEs of AGB-stars. Practically all data have been obtained using the method outlined in Sect. 4, but it should be borne in mind that the data set is relatively heterogeneous, and in many cases the entry applies to a single CSE, that of IRC+10216. The majority of the data comes from Cernicharo et al. (1987a,b), Lindquist et al. (1988, 1992), Sahai \& Wannier (1992), Nyman et al. (1993), Olofsson et al. (1993b), and Omont et al. (1993). The values given should be used with great care. If one is interested in the study of a number of molecules it is advisable to go back to the original data sets. It is notable that, in general, the circumstellar abundance of a molecule is much higher, often by two orders of magnitude, than its interstellar abundance (Cernicharo et al. 1987b). For essentially all species, except $\mathrm{CO}$, the abundance is drastically different in C-rich and $\mathrm{O}$ rich CSEs. In C-rich CSEs the linear carbon-chain molecules dominate, and the abundance decreases only weakly with increasing size of the molecule. However, only slight modifications like the substitution of $\mathrm{CH}_{3}$ for $\mathrm{H}$ lower the abundances substantially, e.g., $\left[\mathrm{CH}_{3} \mathrm{CN}\right] /[\mathrm{HCN}] \approx 4 \times 10^{-4}$ (the interstellar ratio is $\approx 10^{-1}$ ), and other more hydrogen-saturated species like $\mathrm{CH}_{2} \mathrm{CHCN}, \mathrm{CH}_{3} \mathrm{CH}_{2} \mathrm{CN}$, and $\mathrm{CH}_{3} \mathrm{C}_{2} \mathrm{H}$ have not been detected. The abundances of the refractory molecules are low, possibly due to depletion on grains.

The circumstellar molecular abundances in the envelopes of post-AGB objects may differ substantially from those given in Table 2. This will be discussed further in Sect. 8.

\section{Accuracy of estimated circumstellar molecular abundances}

A key question in this connection is the accuracy of the reported molecular abundances. This problem is probably best illuminated by a practical example taken from a study by Olofsson et al. (1990, 1993b). Their sample of carbon stars contains a sub-sample of 30 bright carbon stars whose photospheric characteristics, in particular, the CNO abundances and the effective temperatures, have been reasonably well determined (Lambert et al., 1986). The photospheric HCN, CN, and CS abundances were estimated using the appropriate $\mathrm{CNO}$ abundances (a solar abundance of $S$ was assumed) and effective temperatures in static LTE model atmospheres. The abundances at $\log \tau_{\text {Ross }}=-4$ were adopted. The large spread in carbon excess $[(\mathrm{C} / \mathrm{O}-1)$ ranges from $<0.01$ to 0.8$]$ leads to a relatively large spread in the photospheric abundances (e.g., $6 \times 10^{-6}<f_{\mathrm{HCN}}<5 \times 10^{-5}$ and $10^{-8}<f_{\mathrm{CS}}<3 \times 10^{-5}$ ), which is an advantage in a study of this type.

These stars were observed in radio lines of $\mathrm{CO}, \mathrm{HCN}, \mathrm{CN}$, and $\mathrm{CS}$. The $\mathrm{CO}$ data provided the mass loss rates, i.e., the molecular hydrogen mass loss rate assuming that the photospheric and circumstellar $\mathrm{CO}$ abundance (relative to $\mathrm{H}_{2}$ ) are equal (Olofsson et al. 1993a). The HCN, CN, and CS circumstellar 
Table 2. Molecular abundances in AGB-CSEs

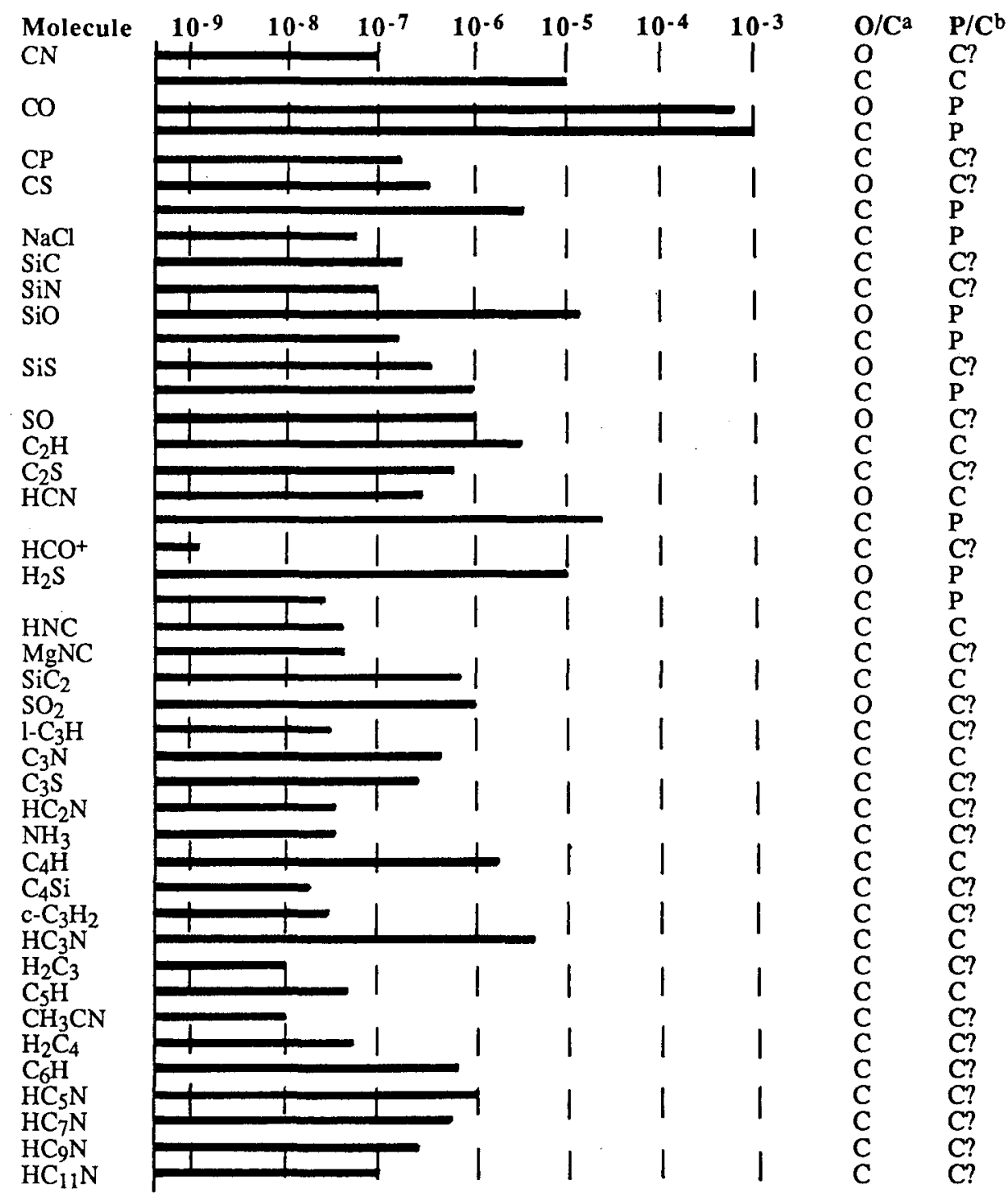

a O-rich or C-rich CSE

b Photospheric or circumstellar origin. ? indicates that no observations exist that conclusively show that the molecule is formed in the CSE. 
abundances were estimated using the model outlined in Sect. 4. It is reasonable to assume that the photospheric and circumstellar abundances of HCN should agree, since there is no known efficient mechanism for producing $\mathrm{HCN}$ in the envelope. The same should apply to CS. Olofsson et al. (1993b) also found a hint of a correlation between the two HCN abundances (after eliminating five somewhat peculiar stars: two ${ }^{13} \mathrm{C}$-rich stars, Y CVn and RY Dra, and three stars whose CSEs may have detached, R Scl, U Cam, and V Hya), but the estimated circumstellar HCN abundances are almost an order of magnitude higher than the corresponding photospheric ones. Olofsson et al. argue that this is due to uncertainties in the circumstellar model, partly based on the fact that the circumstellar CS abundance is also higher than the photospheric one by about the same amount (here the statistics is much worse), but one cannot completely exclude that there is a circumstellar chemistry that affects the abundance in the relatively tenuous and hot CSEs in this sample, and that the photospheric abundances are underestimated (in particular that of $\mathrm{N}$ ). Hence, it seems that even in this relatively well defined problem the estimated circumstellar abundances are off by at least a factor of five.

On the other hand, the results for R Scl and V Hya (and to some extent those for U Cam, Y CVn, and RY Dra) differ so markedly from the expected, that there is every reason to believe that these stars are different from the majority of the stars in the sample, in some way. However, this difference may not be one of elemental composition, but rather a difference in the structure of the CSEs and hence the excitation of the molecules.

It appears that abundance ratios are more reliable than individual abundances. In the case of CN Olofsson et al. (1993b) found circumstellar abundances that are about three orders of magnitude higher than the photospheric ones. This is likely to be much larger than the estimated uncertainties, and hence $\mathrm{CN}$ cannot be a parent molecule. Indeed, the estimated CN/HCN circumstellar abundance ratio is about 0.6 , which is roughly the expected ratio if $\mathrm{CN}$ is formed from photodissociation of $\mathrm{HCN}$.

Another study of interest in this connection is the one by Nyman et al. (1993). They observed the same molecular species in two carbon stars, IRC+10216 and IRAS15194-5115, with similar characteristics, including relatively high mass loss rates of the order a few $10^{-5} \mathrm{M}_{\odot} \mathrm{yr}^{-1}$. The major difference is that the latter source is about five times more distant. Using the model described in Sect. 4, they conclude that for the 13 species observed, the abundances in the two sources agree within a factor of four. A reliable result is also that the ${ }^{12} \mathrm{C} /{ }^{13} \mathrm{C}$-ratio is at least five times lower in IRAS15194-5115 than in IRC +10216 , where the best data suggest a value in the range 40-50 (see Sect. 7.2). This puts some faith to the model, at least as long as the comparison is made within a homogeneous data set. Furthermore, it shows that IRC +10216 is not a unique object; it just happens to be very nearby.

Finally, an illuminating comparison is offered by two recent studies of $\mathrm{SO}$ and $\mathrm{SO}_{2}$ (Sahai \& Wannier, 1992; Omont et al., 1993). For some stars the estimated abundances disagree by a factor of ten between the two studies, and for some 
stars Sahai \& Wannier obtain values that differ by a factor of ten depending on the assumptions made.

\section{Circumstellar molecular line emission: a measure of elemental composition?}

The elemental composition of red giants with very thin CSEs is preferrably estimated using visual and near-IR high-resolution spectra combined with detailed stellar atmosphere models (see e.g., Lambert et al. 1986; Gustafsson 1989; Smith \& Lambert 1990; Lazaro et al. 1991). However, this procedure runs into difficulties as the opacity, and consequently the dust emission, of the CSE increases, and it becomes useless when the mass loss rate is so high that the star is completely obscured. Thus, the elemental composition of the most extreme objects can only be inferred from circumstellar emission, and preferably line emission. However, this is not an easy task since, according to the discussion above, it is difficult to reliably estimate circumstellar molecular abundances. Furthermore, elemental abundances are obtained from these only if the circumstellar chemistry is known. Finally, the circumstellar gas-phase elemental composition may differ appreciably from the stellar atmosphere elemental composition due to selective depletion on grains.

\subsection{The C/O-ratio}

The simplest, and to some extent the most basic, problem would be to distinguish between $\mathrm{O}$-rich and $\mathrm{C}$-rich objects. It is preferable if this can be done using simple line intensity ratios rather than abundance estimates of an individual species. It seems that $\mathrm{SiO}$ and $\mathrm{HCN}$ are the best candidates for this problem if one also takes into account the strength of the lines. In O-rich CSEs we expect $\mathrm{SiO}$ to be abundant while little $\mathrm{HCN}$ should be present since most of the carbon is tied up in CO. In C-rich CSEs we expect the opposite behaviour: $\mathrm{HCN}$ is abundant while little $\mathrm{SiO}$ is present since most of the oxygen is tied up in CO. Both species are presumably radiatively excited except at the highest mass loss rates, and so they should respond in the same manner to different excitation conditions (one potential problem here is that $\mathrm{HCN}$ in $\mathrm{O}$-rich objects is of circumstellar origin). Thus, we expect the $I_{\mathrm{HCN}} / I_{\mathrm{SiO}}$ line intensity ratio to be a strong discriminator between O-rich and C-rich CSEs. Bujarrabal et al. (1993a) and Olofsson et al. (1993c) have recently studied samples of O-rich and $\mathrm{C}$-rich red giants. Olofsson et al. found $I_{\mathrm{HCN}, \mathrm{J}=1-0} / I_{\mathrm{SiO}, \mathrm{v}=0, \mathrm{~J}=2-1 \text {-ratios in }}$ the range $0.05-0.3$ and 3-10 for the $19 \mathrm{O}$-rich objects and the $6 \mathrm{C}-$-rich objects, respectively, where both lines were detected. The results of Bujarrabal et al. agree with this. Thus, the discrimination between the two types of objects is clear and unambiguous.

Next in line stands the question whether a more detailed determination of the $\mathrm{C} / \mathrm{O}-$ ratio can be made. This is a considerably more difficult problem. The effects of trends in the $\mathrm{C} / \mathrm{O}$-ratio are likely to be much smaller than those due 
to whether $\mathrm{C}$ is larger than $\mathrm{O}$ or vice versa. This is because the stability of the $\mathrm{CO}$ molecule, and hence its ability to store $\mathrm{C}$ and $\mathrm{O}$, has such a profound effect on the chemistry.

In view of this we may ask whether the scatter in the $I_{\mathrm{HCN}} / I_{\mathrm{SiO}}$-ratio within the $\mathrm{O}$-rich and $\mathrm{C}$-rich samples discussed above carries any additional information related to the $\mathrm{C}$ and $\mathrm{O}$ abundances. This question cannot as yet be answered. Only marginal detections of $\mathrm{SiO}$ in two carbon stars that belong to the sample of Lambert et al. (1986) have been made (Bujarrabal et al. 1993b) and therefore no comparison between the $I_{\mathrm{HCN}} / I_{\mathrm{SiO}}$ and $\mathrm{C} / \mathrm{O}$ ratios can be made. For the O-rich stars Olofsson et al. (1993c) found that sources with high $F_{25} / F_{12}$ and $F_{60} / F_{25} \mu \mathrm{m}$ flux ratios have only high, in a relative sense, $I_{\mathrm{HCN}} / I_{\mathrm{SiO}}$-ratios. This is interesting since the stars with $2.5 \log F_{60} / F_{25}>-1.8$ fall in a region of the far-IR two-colour diagram of van der Veen \& Habing (1988) that is mainly occupied by carbon stars. On the other hand, the trend of increasing $I_{\mathrm{HCN}} / I_{\mathrm{SiO}}$ with increasing $F_{25} / F_{12} \mu \mathrm{m}$ flux ratio may indicate that it is the mass loss rate that plays the major role. In this connection it is worth noting that Bujarrabal et al. (1993a) found that the S-type stars have line intensity ratios that fall equidistant between those of $\mathrm{O}$-rich and $\mathrm{C}$-rich sources.

Olofsson et al. (1993b) in their study of $\mathrm{HCN}$ in bright carbon stars, also had in mind the possibility of actually determining the $\mathrm{C} / \mathrm{O}$-ratio from circumstellar $\mathrm{HCN}$ and $\mathrm{CO}$ data. The main problem here, apart from the previously discussed difficulty of estimating the $\mathrm{HCN}$ abundance, is that the $\mathrm{CO}$ lines are usually quite optically thick and hence should be relatively insensitive to the $\mathrm{CO}$ abundance. However, models suggest that the mass loss rate scales linearly with the CO line intensity and it is almost inversely proportional to the $\mathrm{CO}$ abundance. If such a relation is used in (2) when estimating the HCN abundance one obtains directly an estimate of the circumstellar HCN/CO abundance ratio. Of course, this estimate is likely to have an associated appreciable uncertainty. Indeed, for the moment it is much larger than acceptable if the aim is to determine the $\mathrm{C} / \mathrm{O}^{-}$ ratio. It is also important to realize that even if the uncertainty in $f_{\mathrm{HCN}} / f_{\mathrm{CO}}$ can be mastered, the problem remains that the relation between $f_{\mathrm{HCN}} / f_{\mathrm{CO}}$ and $\mathrm{C} / \mathrm{O}$ is unique only for low values of $\mathrm{C} / \mathrm{O}$ (for solar abundances of $\mathrm{N}$ and $\mathrm{O}$ this critical value is 0.12 ). For high values of $\mathrm{C} / \mathrm{O}$ the $f_{\mathrm{HCN}} / f_{\mathrm{CO}}$-ratio becomes essentially a measure of the $\mathrm{N}$-abundance, and it has in fact been used for this purpose (Jura, 1991).

Unfortunately, one has to conclude that there is still a long way to go before circumstellar data can be used to obtain detailed information about the elemental composition. However, the reward seems high enough to pursue this research with continued large efforts.

\subsection{Isotope ratios}

Another side of the elemental composition concerns the isotopic ratios of various elements. Here the observational data, i.e., line intensities of the same chemical species but with different isotopic substitutes (isotopomers), are usually more easily converted into astrophysically interesting quantities, the reason being that 
one expects the corresponding lines from the isotopomers to respond in the same way to the physical conditions. In most cases one simply lets line intensity ratios obtained for the isotopomers reflect also the isotope ratios, i.e., ${ }^{\mathrm{n}} \mathrm{X} /{ }^{\mathrm{m}} \mathrm{X}=$ $I\left({ }^{\mathrm{n}} \mathrm{XA}\right) / I\left({ }^{\mathrm{m}} \mathrm{XA}\right)$. Minor corrections for the small differences in beam size and transition probability between the two isotopomers are usually introduced. This method is probably fairly reliable provided (i) that the observed lines from the two isotopomers correspond to the same transition, (ii) that great care is taken in selecting optically thin lines, and (iii) that there is no difference in the spatial distribution and in the excitation of the two isotopomers. To this we shall add that since the lines are weak there is an increased possibility of blending with other lines, or even misidentifications.

In general, the rare isotope lines from CSEs are very weak, and hence the statistics is poor, and, in fact, even worse than in the case of molecular abundances. Only in IRC+10216 does there exist an isotope-ratio setup that is worth discussing. The results for this source obtained by Wannier \& Sahai (1987), Cernicharo \& Guélin (1987), Kahane et al. (1988, 1992), and Wannier et al. (1992) are summarized in Table 3. The uncertainties are of the order $\pm 25 \%$ or less. Major deviations from the terrestrial values are found for $\mathrm{N}\left({ }^{15} \mathrm{~N}\right.$ is highly underabundant) and $\mathrm{O}\left({ }^{18} \mathrm{O}\right.$ is underabundant and ${ }^{17} \mathrm{O}$ is overabundant, and so ${ }^{17} \mathrm{O} /{ }^{18} \mathrm{O}$ is $\approx 8$ times higher than the terrestrial value). Data for ${ }^{15} \mathrm{~N}$ have been obtained in five more C-rich CSEs (Wannier et al. 1992), and for ${ }^{17} \mathrm{O}$ and ${ }^{18} \mathrm{O}$ in four C-rich CSEs (Kahane et al. 1992), but the majority of these sources are post-AGB objects. The results are in line with, but not identical to, those for IRC+10216. These data show the potential, but also the problem that the weakness of the lines severely limits the number of sources that can be studied.

Table 3. Isotope ratios in $\mathrm{IRC}+10216$

\begin{tabular}{lrcl}
\hline Isotope ratio & Ratio & IRC/Solar & Species used \\
\hline${ }^{12} \mathrm{C} /{ }^{13} \mathrm{C}$ & 47 & 0.5 & ${ }^{13} \mathrm{CS}, \mathrm{C}^{34} \mathrm{~S}$ \\
${ }^{14} \mathrm{~N} /{ }^{15} \mathrm{~N}$ & 5300 & 20 & $\mathrm{H}^{13} \mathrm{CN}, \mathrm{HC}^{15} \mathrm{~N}$ \\
${ }^{16} \mathrm{O} /{ }^{17} \mathrm{O}$ & 840 & 0.3 & ${ }^{13} \mathrm{CO}, \mathrm{C}^{17} \mathrm{O}$ \\
${ }^{16} \mathrm{O} /{ }^{18} \mathrm{O}$ & 1300 & 3 & ${ }^{13} \mathrm{CO}, \mathrm{C}^{18} \mathrm{O}$ \\
${ }^{28} \mathrm{Si} /{ }^{29} \mathrm{Si}$ & 19 & 1 & ${ }^{28} \mathrm{SiS},{ }^{29} \mathrm{SiS},{ }^{28} \mathrm{SiC}_{2},{ }^{29} \mathrm{SiC}_{2}$ \\
${ }^{28} \mathrm{Si} /{ }^{30} \mathrm{Si}$ & 29 & 1 & ${ }^{28} \mathrm{SiS},{ }^{30} \mathrm{SiS},{ }^{28} \mathrm{SiC}_{2},{ }^{30} \mathrm{SiC}_{2}$ \\
${ }^{32} \mathrm{~S} /{ }^{33} \mathrm{~S}$ & 100 & 1 & $\mathrm{Si}^{32} \mathrm{~S}, \mathrm{Si}^{33} \mathrm{~S}$ \\
${ }^{32} \mathrm{~S} /{ }^{34} \mathrm{~S}$ & 20 & 1 & $\mathrm{Si}^{32} \mathrm{~S}, \mathrm{Si}^{34} \mathrm{~S}$ \\
${ }^{35} \mathrm{Cl} /{ }^{37} \mathrm{Cl}$ & 2 & 1 & $\mathrm{Na}^{35} \mathrm{Cl}, \mathrm{Na}^{37} \mathrm{Cl}, \mathrm{Al}^{35} \mathrm{Cl}, \mathrm{Al}^{37} \mathrm{Cl}$ \\
\hline
\end{tabular}

In principle, one could expect that the statistics for the ${ }^{12} \mathrm{C} /{ }^{13} \mathrm{C}$-ratio would be relatively good since there is a fair number of detections of ${ }^{12} \mathrm{CO}$ and ${ }^{13} \mathrm{CO}$ in the same objects, and there is some confidence that the modelling of the optically thick ${ }^{12} \mathrm{CO}$ emission is mastered. However, there are two additional problems. Isotope selective photodissociation and fractionation is particularly problematic 
in the case of $\mathrm{CO}$, and the two isotopomers are expected to have different spatial distributions. Furthermore, it is expected that ${ }^{12} \mathrm{CO}$ and ${ }^{13} \mathrm{CO}$ are differently excited. Taken together this results in large uncertainties in the estimates of the ${ }^{12} \mathrm{C} /{ }^{13}$ C-ratio from CO data (Knapp \& Chang 1985; Sopka et al. 1989).

\section{Circumstellar molecular line emission: a measure of stellar evolution?}

In this section we will discuss to what extent there are differences in the appearance of different molecular species as a function of evolutionary stage. Ultimately, it would perhaps be possible to use the appearance and disappearance of various molecular lines to devise a molecular line chronology of stellar evolution on and beyond the AGB. In this we include also the possibility that lines from the same species but with different excitation requirements may contain important information. Since these types of studies are still in their infancies, we will here restrict ourselves to discuss whether there are differences between the "thermal" circumstellar molecular line emission from AGB-stars, post-AGB objects, and planetary nebulae (PNe). Lewis $(1989,1990)$ has made a similar, but much more detailed, study for the strong maser lines.

It should not come as a surprise that the molecular abundances in the CSE around a post-AGB object may differ substantially from those in an AGB-CSE. The reason is twofold. First, the mass loss decreases drastically as the star leaves the AGB, and consequently the CSE detaches from the star. This leads to a rapidly decreasing opacity of the envelope, roughly given by

$$
A_{\mathrm{V}} \approx 20\left(\frac{\dot{M}_{\mathrm{AGB}}}{10^{-6} \mathrm{M}_{\odot} \mathrm{yr}^{-1}}\right)\left(\frac{10 \mathrm{yr}}{t}\right) \mathrm{mag}
$$

where $A_{\mathrm{V}}$ is the visual extinction (the relation $N_{\mathrm{H}_{2}}=10^{21} A_{\mathrm{V} \mathrm{cm}} \mathrm{cm}^{-2} \mathrm{mag}^{-1}$ which seems appropriate for interstellar clouds have been used, Bohlin et al. 1978), and $t$ is the time elapsed since the mass loss terminated. Thus, photodissociation rapidly sets in. Second, eventually the photodissociation increases substantially due to the increasing UV flux from the now much hotter central star. The effect is that the molecular abundances change rapidly as the star leaves the AGB. In general, the abundances decrease, but also new chemical reactions are initiated and the behaviour becomes quite complex. To this we shall add changes in the excitation conditions, which will also have an effect on the molecular line pattern. There is also the possibility that some change in elemental composition may be associated with the termination of the AGB phase.

There is observational evidence that the effects discussed above do lead to changes in the molecular line emission appearance. A nice example is provided by the observations by Bujarrabal et al. (1988) of three C-rich objects in different evolutionary stages, IRC+10216 (AGB-object), CRL2688 (post AGB-object, probably less evolved than CRL618), and CRL618 (post AGB-object, a good pre-PN candidate), (see their Fig. 1). IRC+10216 has been detected in at least 
38 molecular species at radio wavelengths. CRL2688 and CRL618 are still detectable in a fair number of molecular species, 18 and 14 , respectively, and this despite the fact that they are at least a factor of four more distant. Bujarrabal et al. conclude that the molecular abundances in the CSEs of the two latter sources are lower than those in the CSE of IRC+10216, but not drastically so. This is somewhat surprising, but it may indicate that the circumstellar medium is clumped and that therefore the photodissociation is less effective. However, in the young C-rich PN NGC7027, in which the CO lines are still very strong, the number of detections has decreased to five, and the most prominent line, except for those of $\mathrm{CO}$, is one of $\mathrm{HCO}^{+}$(see e.g., Deguchi et al. 1990). Another (probably $\mathrm{C}$-rich) young PN is IRAS21282+5050, it shows detectable $\mathrm{CO}$ and $\mathrm{HCO}^{+}$ but no HCN. This is drastically different to the results for $\mathrm{IRC}+10216$ where the $\mathrm{HCN}$ line is very strong, while the $\mathrm{HCO}^{+}$line is just marginally detected (Lucas \& Guélin 1990). Observations of the more evolved (probably C-rich) PNe NGC2346 (Bachiller et al. 1989) and NGC6072 and IC4406 (Cox et al. 1992) further amplify the situation. $\mathrm{HCO}^{+}$is remarkably strong and it appears that $\mathrm{HCN}$, and in particular $\mathrm{CN}$, regain strength as the object becomes more evolved. We have tried to summarize these findings in Table 4 . Listed are the line intensity ratios $I_{\mathrm{X}, \mathrm{J}=1-0} / I_{\mathrm{CO}, \mathrm{J}=1-0}$, except for $\mathrm{CN}$ and $\mathrm{C}_{2} \mathrm{H}$ where the $N=1-0$ transition has been used, of lines observed with the same telescope. This normalization with respect to the $\mathrm{CO}$ line is not meant to reflect changes in abundance with respect to $\mathrm{CO}$ (the $\mathrm{CO}$ line is normally too optically thick for this), but merely to show the large changes in line intensity for other species than $\mathrm{CO}$, which is known to be easily excited and difficult to destroy. Information on other line intensity ratios is easily extracted from the presented data. Some of the more interesting trends are presented in Fig. 1.

Table 4. Line intensity ratios, $I_{X, 1-0} / I_{C O, 1-0}$, for objects in different evolutionary stages

\begin{tabular}{lclllll}
\hline Source & $\mathrm{HCN}$ & $\mathrm{HNC}$ & $\mathrm{HCO}^{+}$ & $\mathrm{CN}$ & $\mathrm{C}_{2} \mathrm{H}$ & Evolutionary stage \\
\hline IRC+10216 & 0.71 & 0.087 & 0.0015 & 0.89 & 0.16 & AGB \\
CRL2688 & 0.66 & 0.054 & $<0.0075$ & 0.39 & 0.094 & early post-AGB \\
CRL618 & 0.18 & 0.17 & 0.13 & & 0.019 & post-AGB \\
NGC7027 & 0.13 & & 0.30 & 0.04 & $<0.01$ & young PN \\
$21282+5050$ & $<0.005$ & & 0.014 & & & young PN \\
NGC2346 & 0.08 & 0.08 & 0.064 & & & PN \\
NGC6072 & 0.22 & 0.049 & 0.18 & 0.71 & $<0.013$ & PN \\
IC4406 & 0.20 & 0.042 & 0.16 & 0.38 & $<0.015$ & PN \\
\hline
\end{tabular}

The trends are much larger than the uncertainties, and it seems inescapable that most of them are due to photodissociation beyond the AGB and the initiation of a post-AGB circumstellar chemistry (Howe et al. 1992). These results indicate that the molecular line signature of the CSE can be of help in establishing the evolutionary stage of an object. One may also be impressed by the ability 


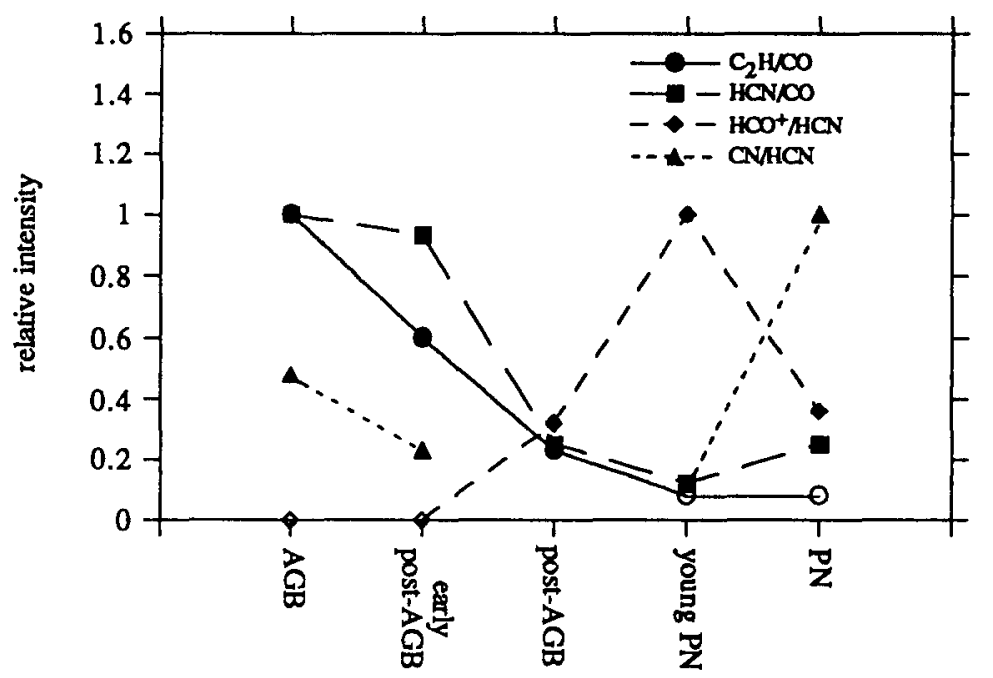

Fig. 1. Line intensity ratios as a function of the evolutionary stage. Open symbols are upper limits. See text for details

of the molecules to survive in even the harshest environments. The results for NGC6072 and IC4406 are astonishingly similar in view of this, and it is probable that there is substantial clumping in the circumstellar medium.

In many post-AGB objects there is evidence for the presence of a highervelocity wind $\left(v_{\mathrm{e}} \approx 50\right.$ to $\left.200 \mathrm{~km} \mathrm{~s}^{-1}\right)$ that may have a bipolar structure rather than a spherical outflow (Olofsson 1993). It would be of great interest to compare the abundances in this wind and the AGB-CSEs. An example of this is provided by the observations of CRL2688 by Jaminet et al. (1992). They claim that the ${ }^{12} \mathrm{C} /{ }^{13} \mathrm{C}$-ratio is four times lower in the fast wind than in the AGB-CSE. If true, this would indicate a change in this isotopic ratio on a time scale of a few hundred years or less. Also the $\mathrm{HCN} / \mathrm{CO}$ abundance ratio appears lower in the fast wind.

\section{Conclusions}

The CSEs of stars on the AGB and beyond show a remarkably rich variety of different molecular species. However, only about a third of them have been detected in more than five sources. The relatively simple geometry and kinematics of the CSEs make it possible to use rather uncomplicated methods for estimating molecular abundances that are accurate to within a factor of about five or slightly worse in some cases. The methods have not yet reached the level of sophistication that allows the circumstellar data to be used to obtain detailed information about the the elemental composition. However, the results on isotope ratios are already of high quality. It is clear that different molecular species 
behave differently as the object evolves, and there is good hope that one may eventually be able to use this as a kind of molecular line chronology for stellar evolution on and beyond the AGB.

There remains much to be done in the improvement of the circumstellar models used. In particular, a detailed non-LTE, non-local radiative transfer analysis is required, but for the majority of the molecules the spectroscopic data and the collisional cross sections are lacking. Another important quantity for which the uncertainty must be considerably lowered is the mass loss rate. Also, the actual distribution of matter in a CSE, e.g., to what extent is the material clumped, may have a profound effect on the resulting abundance estimates.

Acknowledgements. I am grateful to the Knut and Alice Wallenberg foundation and the organizers of IAU Colloquium 146 for travel support. I also value the comments on the manuscript by John Black. Finally, I am grateful to Per Bergman for his support in the transformation of my manuscript into a Latexfile.

\section{References}

Alcolea J., Bujarrabal V., 1992, Astron. Astrophys., 253, 475

Bachiller R., Bujarrabal V., Martín-Pintado J., Gómez-González J., 1989, Astron. Astrophys., 218, 252

Bergman P., Carlström U., Olofsson H., 1993, Astron. Astrophys., 268, 685

Betz A., 1987, in Astrochemistry, IAU Symp. No. 120, eds M.S. Vardya and S.P. Tarafalar, Reidel, Dordrecht, p.327

Bieging J.H., Chapman B., Welch W.J., 1984, Astrophys. J., 285, 656

Bieging J.H., Nguyen-Q-Rieu, 1988, Astrophys. J., 329, L107

Bieging J.H., Nguyen-Q-Rieu, 1989, Astrophys. J., 343, L25

Bieging J.H., Tafalla M., 1993, Astron. J., 105, 576

Bohlin R.C., Savage B.D., Drake J.F., 1978, Astrophys. J., 224, 132

Bowers P.F., Johnston K.J., Spencer J.H., 1983, Astrophys. J., 274, 733

Bowers P.F., Reid M.J., Johnston K.J., Spencer J.H., Moran J.M., 1980, Astrophys. $J ., 242,1088$

Bujarrabal V., Fuente A., Omont A., 1993a, Astron. Astrophys., subm.

Bujarrabal V., Fuente A., Omont A., 1993b, Astron. Astrophys., subm.

Bujarrabal V., Gómez-González J., Bachiller R., Martín-Pintado J., 1988, Astron. Astrophys., 204, 242

Carlström U., Olofsson H., Johansson L.E.B., Nguyen-Q-Rieu, Sahai R., 1990, in From Miras to Planetary Nebulae - Which Path for Stellar Evolution, eds M.O. Mennessier and A. Omont, Editions-Frontiérs, p.170

Cernicharo J., Bujarrabal V., 1992, Astrophys. J., 401, L109

Cernicharo J., Bujarrabal V., Santarén J.L., 1993, Astrophys. J., 407, L33

Cernicharo J., Guélin M., 1987, Astron. Astrophys., 183, L10

Cernicharo J., Guélin M., Hein H., Kahane C., 1987a, Astron. Astrophys., 181, L9

Cernicharo J., Guélin M., Menten K.M., Walmsley C.M., 1987b, Astron. Astrophys., 181, L1

Cernicharo J., Thum C., Hein H., John D., Garcia P., Mattioco F., 1990, Astron. Astrophys., 231, L15 
Clegg R.E.S., Hinkle K.H., Lambert D.L., 1982, MNRAS, 201; 95

Cohen R.J., 1989, Reports on Progress in Physics, 52, 881

Cox P., Omont A., Huggins P.J., Bachiller R., Forveille T., 1992, Astron. Astrophys., 266,420

Dayal A., Bieging J.H., 1993, Astrophys. J., 407, L37

Deguchi S., Izumiura H., Kaifu N., Mao X., Nguyen-Q-Rieu, Ukita N., 1990, Astrophys. $J ., 351,522$

Elitzur M., 1992, Astronomical Masers, Kluwer, Dordrecht

Gensheimer P.D., Likkel L., Snyder L., 1992, Astrophys. J., 388, L31

Glassgold A.E., Huggins P.J., 1983, MNRAS, 203, 517

Glassgold A.E., Omont A., Guélin M., 1992, Astrophys. J., 396, 115

Guilloteau S., Omont A., Lucas R., 1987, Astron. Astrophys., 176, L24

Gustafsson B., 1989, Ann. Rev. Astr. Astroph., 27, 701

Howe D.A., Millar T.J., 1990, MNRAS, 244, 444

Howe D.A., Millar T.J., Williams D.A., 1992, MNRAS, 255, 217

Huggins P.J., Glassgold A.E., 1982, Astrophys. J., 252, 201

Izumiura H.,1990, PhD thesis, Tokyo University

Izumiura H., Ukita N., Kawabe R., Kaifu N., Tsuji T., Unno W., Koyama K., 1987, Astrophys. J., 323, L81

Jaminet P.A., Danchi W.C., Sandell G., Sutton E.C., 1992, Astrophys. J., 400, 535

Jewell P.R., Snyder L.E., Walmsley C.M., Wilson T.L., 1991, Astron. Astrophys., 242, 211

Jura M., 1991, A strophys. J., 372, 208

Jura M., Morris M., 1981, Astrophys. J., 251, 181

Kahane C., Gómez-González J., Cernicharo J., Guélin M. , 1988, Astron. Astrophys., 190, 167

Kahane C., Cernicharo J., Gómez-González J., Guélin M.:, 1992, Astron. Astrophys., 256, 235

Kawaguchi K., Kagi E., Hirano T., Takano S., Saito S. , 1993, Astrophys. J., 406, L39

Knapp G.R., Chang K.M., 1985, Astrophys. J., 293, 281

Lambert D.L, Gustafsson B., Eriksson K., Hinkle K.H., 1986, Astrophys. J. Suppl., 62, 373

Lazaro C., Lynas-Gray A.E., Clegg R.E.S., Mountain C.M., Zadrozny A., 1991, MNRAS, 249, 62

Lewis B.M., 1989, Astrophys. J., 338, 234

Lewis B.M., 1990, Astron. J., 99, 710

Lewis B.M., Engels D., 1991, MNRAS, 251, 391

Lindquist M., Nyman L.- $\AA$., Olofsson H., Winnberg A., 1988, Astron. Astrophys., 205, L1

Lindqvist M., Olofsson H., Winnberg A., Nyman L.- $\AA ., 1992$, Astron. Astrophys., 263, 183

Lucas R., Cernicharo J., 1989, Astron. Astrophys., 218, L20

Lucas R., Guélin M., 1990, in Submillimetre Astronomy, eds G.D. Watt and A.S. Webster, Kluwer, Dordrecht, p.97

Lucas R., et al., 1992, Astron. Astrophys., 262, 491

Mamon G.A., Glassgold A.E., Huggins P.J., 1988, Astrophys. J., 328, 797

Menten K.M., Melnick G.J., 1989, Astrophys. J., 341, L91

Menten K.M., Melnick G.J., 1991, Astrophys. J., 377, 647

Menten K.M., Melnick G.J., Phillips T.G., 1990, Astrophys. J., 350, L41 
Millar T.J., 1988, in Rate Coefficients in Astrochemistry, eds T.J. Millar and D.A. Williams, Kluwer, Dordrecht, p. 287

Nejad L., Millar T.J., 1987, Astron. Astrophys., 183, 279

Nejad L., Millar T.J., 1988, MNRAS, 230, 79

Nercessian E., Guilloteau S., Omont A., Benayoun J.J., 1989, Astron. Astrophys., 210, 225

Nguyen-Q-Rieu, Bujarrabal V., Olofsson H., Johansson L.E.B., Turner B.E., 1984, Astrophys. J., 286, 276

Nyman L.- $\AA$., Olofsson H., 1985, Astron. Astrophys., 147, 309

Nyman L.-A., Olofsson H., Johansson L.E.B., Booth R.S., Carlström U., Wolstencroft R., 1993, Astron. Astrophys., 269, 373

Olofsson H.: 1993, in Mass Loss on the $A G B$ and Beyond, ed. H. Schwarz, in press

Olofsson H., Carlström U., Eriksson K., Gustafsson B., 1992, Astron. Astrophys., 253, L17

Olofsson H., Eriksson K., Gustafsson B., Carlström U., 1993a, Astrophys. J. Suppl., 87, 267

Olofsson H., Eriksson K., Gustafsson B., Carlström U., 1993b, Astrophys. J. Suppl., 87, 305

Olofsson H., Lindqvist M., Winnberg A., Nyman L.- $\AA ., 1993 c$, in prep.

Omont A., Lucas R., Morris M., Guilloteau S., 1993, Astron. Astrophys., 267, 490

Sahai R., Bieging J.H., 1993, Astron. J., 105, 595

Sahai R., Wannier P.G., 1992, Astrophys. J., 394, 320

Scalo J.M., Slavsky D.B., 1980, Astrophys. J., 239, L73

Smith V.V., Lambert D., 1990, Astrophys. J. Suppl., 72, 387

Solomon P., Jefferts K.B., Penzias A.A., Wilson R.W., 1971, Astrophys. J., 163, L53

Sopka R.J., Olofsson H., Johansson L.E.B., Nguyen-Q-Rieu, Zuckerman B., 1989, Astron. Astrophys., 210, 78

Takano S., Saito S., Tsuji T., 1992, PASJ, 44, 469

Truong-Bach, Nguyen-Q-Rieu, 1989, Astron. Astrophys., 214, 267

Truong-Bach, Morris D., Nguyen-Q-Rieu, 1992, Astron. Astrophys., 249, 435

Truong-Bach, Morris D., Nguyen-Q-Rieu, Deguchi S., 1990, Astron. Astrophys., 230, 431

Turner B.E., 1987a, Astron. Astrophys., 182, L15

Turner B.E., 1987b, Astron. Astrophys., 183, L23

van der Veen W.E.C.J., Habing H.J., 1988, Astron. Astrophys., 194, 125

van der Veen W.E.C.J., Olofsson H., 1990, in From Miras to Planetary Nebulae - Which Path for stellar Evolution, eds M.O. Mennessier and A. Omont, Editions-Frontiérs, p. 139

van Dishoeck E.F., 1988, in Rate Coefficients in Astrochemistry, eds T.J. Millar and D.A. Williams, Kluwer, Dordrecht, p. 49

Wannier P., Sahai R., 1987, Astrophys. J., 319, 367

Wannier P. Andersson B.-G., Olofsson H., Ukita N., Young K., 1992, Astrophys. J., 380, 593

Wilson W.J., Barrett A.H., 1968, Science, 161, 778 